\title{
Avaliação dos ruídos em uma unidade neonatal de um hospital universitário
}

\section{Evaluation of noise in a neonatal unit at a university hospital}

\author{
Ariadne Pinheiro Nazario'; Vivian Carolina Benetti Jacinto Santos ${ }^{2}$; Edilaine \\ Giovanini Rossetto ${ }^{3}$; Sarah Nancy Degau Hegeto de Souza ${ }^{4}$; Nelma Ellen \\ Zamberlan Amorim ${ }^{5}$; Carmen Gracinda Silvan Scochi ${ }^{6}$
}

\section{Resumo}

Objetivo: Identificar e mensurar as principais fontes de ruído, dimensionar os níveis de ruído no ambiente e conhecer a percepção dos funcionários acerca dos ruídos em uma Unidade Neonatal.

Material e método: Estudo quantitativo, desenvolvido na Unidade Neonatal de um Hospital Universitário no norte do Paraná, em três etapas: levantamento das principais fontes de ruído e subsequente mensuração; entrevista com a equipe multiprofissional relacionada à percepção dos mesmos acerca dos ruídos na unidade; e dimensionamento do ruído no ambiente por duas semanas não consecutivas.

Resultados: As principais fontes ruidosas encontradas foram: alarmes de monitores e equipamentos, torneira (aberta), tampa da lixeira, entre outros. A média das duas semanas foi: Leq 44,4 dB, Lmax 104,5 dB, Lmin 40 dB e Lpeak de 144,8 dB. Os funcionários reconheceram que o ruído intenso acarreta prejuízo a eles e aos bebês. Conclusão: Constatou-se que o valor médio dos ruídos está dentro do preconizado, porém com níveis altíssimos de Lpeak e Lmax.

Palavras-chave: Ruído. Unidades de terapia intensiva neonatal. Prematuro. Enfermagem neonatal.

\begin{abstract}
Objective: To identify and measure the main sources of noise, to determine the levels of ambient noise and to know the perception of employees about noise in a Neonatal Unit.

Material and method: A quantitative study developed in a Neonatal Unit School Hospital, Paraná, Brazil, conducted in three steps: identification of the main sources of noise and subsequent measure; interview with the multidisciplinary team about the perception of noise in the unit; and dimensioning noise ambient for two non-consecutive weeks.

Results: The main sources of noise were: monitor and equipment alarms, open water faucets, trash can lids, among others. The mean) of the two weeks period was: Leq $44.4 \mathrm{~dB}$, Lmax $104.5 \mathrm{~dB}$, Lmin $40 \mathrm{~dB}$ and Lpeak $144.8 \mathrm{~dB}$. Employees acknowledged that intense noise is prejudicial to them and to babies.

Conclusion: It was found that the mean value of the noise is within the recommended, but with very high levels of Lpeak and Lmax.
\end{abstract}

Keywords: Noise. Neonatal intensive care units. Premature infant. Neonatal nursing.

${ }^{1}$ Enfermeira Especialista em Enfermagem Neonatal pela modalidade Residência em Enfermagem Neonatal da Universidade Estadual de Londrina (UEL) - PR.

${ }^{2}$ Enfermeira Especialista em Enfermagem Neonatal pela modalidade Residência em Enfermagem Neonatal da Universidade Estadual de Londrina (UEL) - PR.

${ }^{3}$ Enfermeira. Doutora em Saúde Pública pela Escola de Enfermagem de Ribeirão Preto da Universidade de São Paulo (EERP/ USP). Professora do Departamento de Enfermagem da Universidade Estadual de Londrina (UEL) - PR.

${ }^{4}$ Enfermeira. Doutora em Saúde Pública pela Escola de Enfermagem de Ribeirão Preto da Universidade de São Paulo (EERP/ USP). Professora do Departamento de Enfermagem da Universidade Estadual de Londrina (UEL) - PR.

${ }^{5}$ Fonoaudióloga. Doutora em Enfermagem em Saúde Pública da Escola de Enfermagem de Ribeirão Preto da Universidade de São Paulo (EERP/USP).

${ }^{6}$ Enfermeira. Professora Titular do Departamento de Enfermagem Materno-Infantil e Saúde Pública da EERP/USP. 


\section{Introdução}

Nas últimas décadas, o cuidado nas Unidades de Terapia Intensiva Neonatal (UTIN) e Unidade de Cuidado Intermediário Neonatal (UCIN) tem recebido melhorias significativas, tanto do ponto de vista tecnológico, quanto da veiculação de evidências científicas e humanização do cuidado. Apesar da importância dessas transformações para o tratamento do Recém-Nascido (RN), em muitas destas Unidades Neonatais (UNs) não existe a preocupação necessária com as peculiaridades de cada RN e seu desenvolvimento integral (GAÍVA; MARQUESI; ROSA, 2010; SANTOS; RIBEIRO; SANTANA, 2012).

O ambiente da UTIN é estressante (RODARTE et al., 2005) e superestimulante, muitas vezes gera desconforto e dor ao RN (SANTOS; RIBEIRO; SANTANA, 2012), muito diferente do ambiente intrauterino no qual o bebê estava acostumado. $\mathrm{O}$ ambiente intrauterino é tranquilo, silencioso, escuro e aconchegante, e quando o bebê nasce antes do tempo, ele depara-se com um ambiente onde há luminosidade e ruídos em excesso, circulação constante de pessoas e interrupções do ciclo de sono e vigília (REICHERT; LINS; COLLET, 2007).

Estudos afirmam que a UN pode causar danos ao desenvolvimento neuromotor dos bebês que necessitam de internação, principalmente os RNs prematuros, já que estes passam um tempo prolongado de internação e têm o cérebro ainda em desenvolvimento (TAMEZ; SILVA, 2013).

O sistema nervoso central dos RNs a termo tem a vantagem de possuir maior maturidade com melhor habilidade para adaptação e ajustamento ao ambiente extrauterino. Em relação aos prematuros, que são mais susceptíveis aos efeitos do meio ambiente, quanto menor a idade gestacional, maior o comprometimento, pois o desenvolvimento cerebral ainda não está completo, o que aumenta o risco de maturação cerebral anormal (TAMEZ; SILVA, 2013).

No Brasil, somente na década de 90 é que iniciaram os estudos direcionados à questão do ruído em ambiente neonatal (RODARTE et al., 2005). Os níveis intensos de ruídos presentes nas UNs podem causar sérios danos ao desenvolvimento e crescimento dos bebês prematuros e (de) baixo peso, que quando superestimulados apresentam estresse, percebido através de vários sinais, tais como: respiração irregular, apneia, diminuição da oxigenação, aumento da frequência cardíaca e respiratória, palidez, cianose, náusea, vômitos, eructação, flacidez, tremores, irritabilidade e choro (TAMEZ; SILVA, 2013).

Segundo a Associação Brasileira de Normas Técnicas (ABNT), por meio da NBR10152, os níveis de ruído esperados em um ambiente hospitalar são de 30 a 55 decibéis (dB) (ASSOCIAÇÃO BRASILEIRA DE NORMAS TÉCNICAS, 1987). No entanto, para a UN este valor é mais restrito, devendo permanecer na faixa de $35 \mathrm{~dB}$, sendo este o nível sonoro para conforto acústico, e $45 \mathrm{~dB}$, valor aceitável (ASSOCIAÇÃO BRASILEIRA DE NORMAS TÉCNICAS, 1987).

$\mathrm{Na}$ década de 1980, Als desenvolveu uma modalidade de proteção ao desenvolvimento dos RNs pré-termo, denominada Neonatal Individualized Developmental Care and Assessment Program (NIDCAP), que visa modificar positivamente o ambiente das UTINs (ALS, 1982). O cuidado desenvolvimental é composto por algumas áreas de cuidado, sendo elas o manejo do ambiente (diminuição do ruído e da luminosidade), o posicionamento do bebê em flexão (simulando a posição intrauterina), agrupamento dos cuidados (evitando o excesso de manipulação), a sucção não nutritiva, o cuidado canguru e incentivo ao aleitamento materno, as atividades de promoção de autorregulação dos estados de consciência e a participação dos pais no cuidado, na perspectiva do cuidado centrado na família (ALS, 1982; BYERS, 2003).

Com base nos resultados de varias pesquisas (AURÉLIO; TOCHETTO, 2010a; KAKEHASHI et al., 2007; PEIXOTO et al., 2011; ZAMBERLAN et al., 2008) que encontraram níveis de ruídos acima do 
desejado nesta $\mathrm{UN}$ e o interesse especial nessa área do cuidado desenvolvimental que visa o controle e diminuição dos ruídos na UTIN, surgiu a seguinte questão de pesquisa: como está a UN em questão em relação à produção de ruídos? Assemelha-se à maioria dos estudos disponíveis na literatura? O que seria necessário para o aprimoramento do ambiente e melhor desenvolvimento dos bebês?

Considerando que a ausência de controle de ruídos, bem como a identificação das suas principais fontes nas UNs faz-se necessário conhecer esses dados com a finalidade de evitar os potenciais danos provocados aos RNs internados por longos períodos. Os objetivos deste estudo foram identificar as principais fontes de ruídos no ambiente de uma UN, dimensionar os níveis de ruídos e conhecer a percepção dos trabalhadores acerca dos ruídos de uma UN de um hospital universitário.

\section{Material e Método}

Foi realizado um estudo quantitativo, observacional e descritivo, desenvolvido em uma UN, que contempla leitos de UTIN e UCIN de um hospital universitário no sul do país. Trata-se um hospital público de alta complexidade, que possui atendimento pelo Sistema Único de Saúde (SUS) em sua totalidade. A UN é dividida em leitos de UTIN e UCIN, correspondendo a sete leitos intensivos e dez de cuidados intermediários. Constitui um Hospital Amigo da Criança, referência para prematuros de alto risco em toda a região norte do Paraná. Os pais podem permanecer diariamente na $\mathrm{UN}$ todo o tempo que puderem e desejarem.

O estudo foi desenvolvido em três etapas, sendo que na primeira realizou-se a caracterização das principais fontes de ruídos presentes na UCIN, como jato d'água da torneira, tampa da lixeira, alarmes de incubadoras, oxímetros e ar condicionado, para posterior mensuração das mesmas. Foram avaliados equipamentos com a mesma finalidade e de marcas diferentes. AUCIN foi escolhida por ser a unidade onde os bebês encontravam-se mais estáveis, possibilitando assim manter o ambiente constante durante toda a coleta, com o cuidado de não haver interferências nos resultados. Foi utilizado um decibelímetro modelo Quest Technologies Sound Pro SE/DL, sendo verificada sua eficácia através da calibração antes do uso.

As fontes de ruído foram mensuradas com o decibelímetro a uma distância de $30 \mathrm{~cm}$ por um período de três minutos consecutivos para avaliar a variação de decibéis. Este método de medição foi determinado após orientações e treinamento das pesquisadoras, fornecidos por pesquisadora do assunto, com experiências prévias com este tipo de metodologia e utilização destes equipamentos (ZAMBERLAN, 2006; ZAMBERLAN et al., 2008).

Para as fontes explicadas a seguir, não houve necessidade da permanência dos três minutos, com o intuito de diminuir a exposição dos pacientes a esses procedimentos e também o desperdício de materiais. A abertura e fechamento da portinhola da incubadora, o abrir e fechar as portas de madeira e de acrílico de correr foram avaliadas por cinco vezes, enquanto a retirada de papéis do dispensador utilizou-se o tempo de observação de um minuto, o que foi suficiente para a análise do estudo.

Para a mensuração do ruído ocorrido com a tampa do lixo, a mesma foi aberta 25 vezes e para o ruído com o deslocamento da mesa de inox com rodas para suporte de materiais, foi necessário que ela fosse transportada pelo centro da unidade por duas vezes consecutivas. Durante as medições dos ruídos provocados para coleta de dados, os bebês receberam um protetor auditivo para evitar desconforto e prejuízos pela exposição aos ruídos produzidos.

$\mathrm{Na}$ segunda etapa, realizou-se entrevistas com a equipe multiprofissional em relação à percepção dos funcionários da UN acerca dos ruídos presentes na unidade. Todos os profissionais foram convidados e sua participação foi voluntária, não havendo critérios de exclusão de participantes. A entrevista foi realizada somente após a assinatura o Termo de Consentimento Livre e Esclarecido. 
A opinião dos entrevistados foi obtida por meio das seguintes questões: Na sua opinião, o que é ruído? Você acha que o ruído presente em sua UN incomoda? Se sim, quanto incomoda? Em que o ruído mais interfere na sua rotina de trabalho? Qual local da UN você acha o ruído mais intenso? Fale sobre os efeitos da exposição ao barulho ambiente para os RNs, seus acompanhantes e profissionais que atuam na UN. Vocês consideram que esta unidade é barulhenta? Quais as fontes de ruído intenso? O que você sugere para diminuir o ruído dentro da sua UN?

$\mathrm{Na}$ terceira etapa realizou-se o dimensionamento do ruído com a utilização do dosímetro modelo Quest 400, instalado no centro da UTIN. O instrumento integraliza o nível sonoro a cada minuto e armazena 999 eventos separadamente na memória, apresentando uma tela de visualização que permite eleger os múltiplos parâmetros (dose acumulada) ou um só parâmetro amplificado. Foi utilizado como padrão de referência o nível de pressão sonora (NPS). Foram realizados cálculos de Leq (energia equivalente do nível de pressão), que é o nível médio de energia sonora medido em dB em um determinado tempo; Lmax, picos de energia acústica, de duração menor que um segundo, ocorrendo em intervalos superiores a um segundo; Lpeak, é o mais alto nível de pressão sonora instantâneo registrado e Lmin, que é o menor nível de energia acústica registrado. O equipamento foi programado para operar em intervalos de NPS entre 40 e $140 \mathrm{~dB}$, a fim de se obter o menor valor registrado pelo equipamento e o máximo permitido para o adulto. O limite para crianças é de $120 \mathrm{~dB}$ (WORLD HEALTH ORGANIZATION, 1999).

Após a fixação do aparelho no centro da unidade, seguiu-se o recomendado para medir o ruído ambiental, que consiste na colocação do microfone do dosímetro equidistante a um metro de quaisquer superfícies, como paredes, teto, piso e móveis (ASSOCIAÇÃO BRASILEIRA DE NORMAS TÉCNICAS, 2000). Porém, devido a este um metro de distância preconizada entre o aparelho e o teto influenciar no processo de trabalho do setor, houve a necessidade de adaptação para $80 \mathrm{~cm}$ de distância.
Foi realizado dimensionamento em duas semanas não consecutivas, a primeira de 17 a 23/12/2011 e segunda de 20 a 26/01/2012. Antes de cada coleta foi feito uma operação de dessensibilização da equipe, quando o dosímetro foi pendurado na unidade como se estivesse ligado, simulando as trocas de bateria, com o intuito de diminuir o impacto da presença do equipamento no ambiente para monitoramento.

$\mathrm{Na}$ primeira semana, os dados foram coletados continuamente das $07 \mathrm{~h}$ às $19 \mathrm{~h}$ e a troca de baterias foi feita entre 20h30minh e $21 \mathrm{~h}$, diariamente. Durante a segunda semana, os dados foram coletados das $19 \mathrm{~h}$ às $07 \mathrm{~h}$, e a troca de baterias feita entre as $15 \mathrm{~h}$ e $16 \mathrm{~h}$, diariamente. Durante o período de coleta matutino, uma das pesquisadoras elaborou um diário de campo para observação das fontes intensas, para posterior análise das alterações.

Os dados obtidos no Quest400 foram descarregados para o programa QuestSuitMR para Windows $®$ sendo possível processar os NPS em respostas numéricas e transportar em planilhas do Microsoft Excel 2010®. Os dados foram comparados de acordo com as normas nacionais, internacionais e com a literatura.

O projeto de pesquisa foi aprovado pelo Comitê de Ética em Pesquisa Envolvendo Seres Humanos da Universidade Estadual de Londrina (CEP/UEL), sob o parecer 181/2011, CAAE n ${ }^{\circ}$ 0124.0.268.268-11.

\section{Resultados}

No corrente estudo foram identificadas as principais fontes de ruídos presentes na UN. Para escolha das mesmas, foi realizada uma observação da rotina da unidade. Após esse elenco dessas, foi escolhido um dia para medição pontual dos níveis dos ruídos das fontes observadas. No dia estabelecido, permaneceram dentro do ambiente da UCIN duas funcionárias e duas pesquisadoras, ar condicionado (ligado) e lâmpadas (acesas), cinco incubadoras ligadas, quatro bebês internados, um respirador funcionando, quatro oxímetros de pulso em uso, quatro bombas de infusão 
em em funcionamento e as duas portas de acesso, uma de madeira e outra de acrílico, as quais permaneceram fechadas. Havia conhecimento prévio da pesquisa por parte de todos os funcionários, com a finalidade de evitar interrupções no processo de medição.

A Figura 1 mostra em ordem decrescente os valores encontrados, em decibéis.

Figura 1 - Fontes de ruídos avaliadas na UCIN, em decibéis. Londrina, 2012.

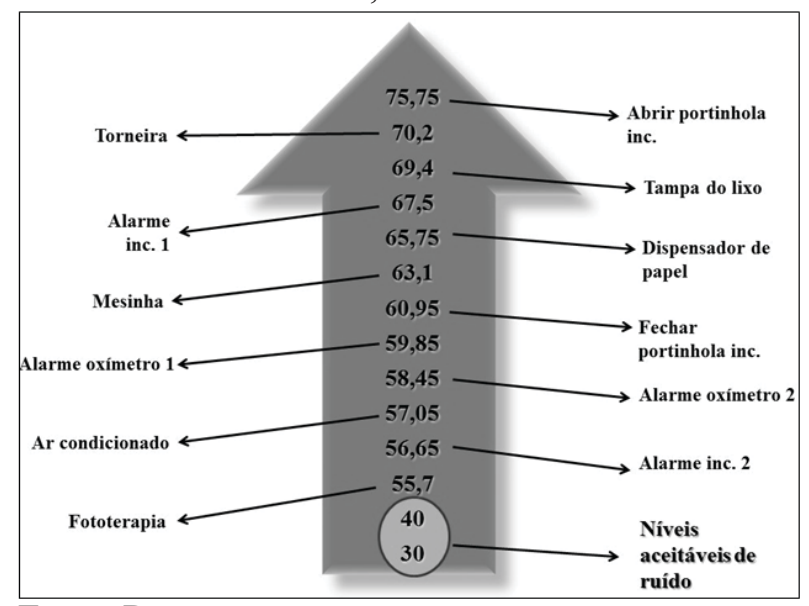

Fonte: Do autor.

Foram entrevistadas 17 profissionais, sendo 15 auxiliares/técnicas de enfermagem, uma enfermeira e uma fisioterapeuta, com o intuito de conhecer a percepção destas acerca dos ruídos na UN.

Observa-se na Tabela 1 que os alarmes dos aparelhos, seguido das conversas paralelas, foram as principais fontes de ruído apontadas pelos profissionais. $\mathrm{O}$ aparecimento frequente das fontes nas falas dos profissionais, evidencia que eles reconheceram as alterações auditivas, a irritabilidade, o estresse e as alterações no ciclo se sono e vigília como os principais efeitos do ruído para os RNs.

Dos 17 profissionais que responderam ao questionário, $15(88,2 \%)$ consideraram a unidade barulhenta e incomodam-se com o barulho em excesso. Os profissionais também apontaram alguns efeitos que os ruídos provocavam na sua rotina de trabalho, tais como diminuição da concentração, irritabilidade e estresse, distração, agitação e alterações auditivas, em ordem de frequência de aparecimento nas falas.

Os profissionais que responderam ao questionário apontaram algumas sugestões para melhor controle dos ruídos dentro da UN como: manutenção periódica dos aparelhos (incubadoras, oxímetros), conscientização da equipe, abaixar tom de voz, mudanças nas lixeiras, diminuir o volume dos alarmes, minimizar o acesso à unidade, local próprio para lavagem de incubadora, manter as rodas dos objetos emborrachadas, ajustar o barulho da torneira, não ligar a umidade quando o reservatório estiver sem água.

$\mathrm{Na}$ mensuração continua dos níveis de pressão sonora, durante duas semanas não consecutivas, sendo a primeira no período diurno e a segunda no período noturno, obteve-se um total de 168 horas de coleta. Destas 168 horas, o valor médio de energia equivalente $\left(\mathrm{L}_{\mathrm{eq}}\right)$ ao nível de pressão sonora medido em decibéis foi de $44,4 \mathrm{~dB}$, o pico de energia acústica $\left(\mathrm{L}_{\max }\right)$ foi de 104,5 dB, o menor nível de energia acústica $\left(\mathrm{L}_{\min }\right)$ foi de $40 \mathrm{~dB}$ e o nível mais alto de pressão sonora instantânea $\left(\mathrm{L}_{\text {peak }}\right)$ foi de $144,8 \mathrm{~dB}$.

Tabela 1 - Principais fontes de ruído e seus efeitos para os RNs apontados pelos profissionais da UN. Londrina, 2012

\begin{tabular}{lrrllr}
\hline \multicolumn{1}{c}{ Eontes de ruídos } & n & \% & & Efeitos dos ruídos & \% \\
\hline Alarme de aparelhos & 13 & 33 & Alterações auditivas & 7 & 29 \\
Conversas paralelas & 9 & 23 & Irritabilidade e estresse & 6 & 25 \\
Telefone & 5 & 13 & Alterações no ciclo de sono e vigília & 6 & 25 \\
Lixeiras & 4 & 10 & Desconforto & 2 & 8 \\
Objetos sendo arrastados & 2 & 5 & Taquicardia & 1 \\
Intercorrências & 2 & 5 & Hiperatividade & 4 \\
Fluxo intenso de pessoas & 1 & 3 & Distúrbios comportamentais a longo prazo & 1 \\
Gaveta do carrinho de emergência & 1 & 3 & & 4 \\
Lavagem da incubadora & 1 & 3 & & \\
Válvula redutora de oxigênio & 1 & 3 & & \\
Portinhola da incubadora & 1 & 3 & &
\end{tabular}

Fonte: Do autor. 
Em relação à mensuração semanal do ruído no ambiente da UTIN, os valores diários obtidos encontram-se nos gráficos abaixo, sendo o período diurno exposto no Gráfico 1, e o período noturno, no Gráfico 2. Não foi possível mensurar um nível mínimo de ruído menor que $40 \mathrm{~dB}$, já que o equipamento foi programado para registrar o NPS entre os intervalos de 40 e $140 \mathrm{~dB}$, porém é possível perceber em todos os dias de coleta dos dados (diurno e noturno) que este nível mínimo estabelecido, ou menor, foi atingido, o que sugere que pelo menos por algum período do tempo de medição os níveis ideais de NPS foram encontrados. Entretanto, o pico máximo de $\operatorname{NPS}\left(\mathrm{L}_{\text {peak }}\right)$ ocorreu em três dias consecutivos no período diurno, enquanto não se observou comportamento semelhante no noturno.

Gráfico 1 - Níveis de ruídos observados durante o período diurno de coleta na UTIN, em decibéis. Londrina, 2012.

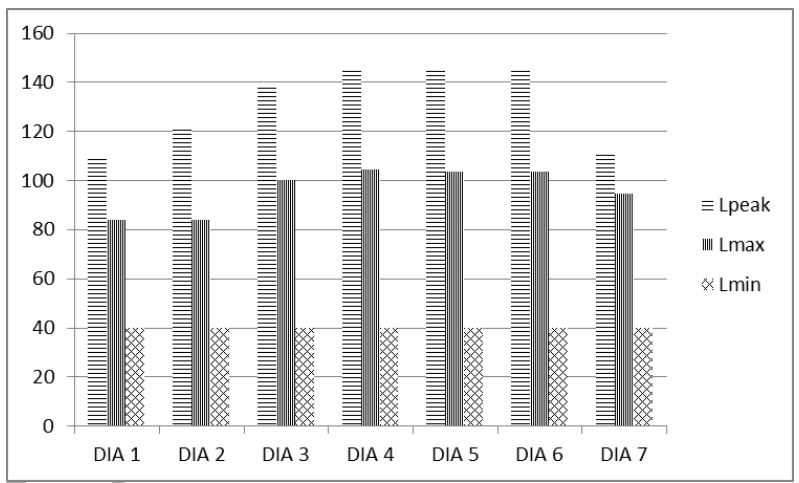

Fonte: Do autor.

Gráfico 2 - Níveis de ruídos observados durante o período noturno de coleta na UTIN, em decibéis. Londrina, 2012.

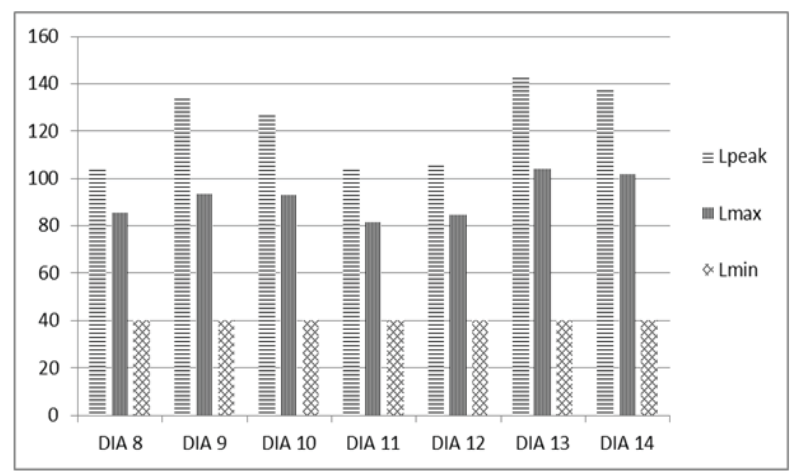

Fonte: Do autor.
Após o término da coleta de dados, os resultados obtidos foram apresentados à equipe da UN em forma de discussão participativa. Foram debatidas as respostas obtidas e as sugestões para um melhor controle de ruídos.

\section{Discussão}

Os níveis de pressão sonora obtidos com o dimensionamento das fontes pontuais de ruídos mostraram que os RNs estão expostos constantemente a níveis muito acima do recomendado pela ABNT. Os alarmes dos equipamentos, necessários para monitorização constante do bebê, a torneira, indispensável para a lavagem das mãos, a lixeira, enfim, objetos necessários para o bom funcionamento da unidade, porém, com altos níveis de ruídos, podem causar todas as alterações fisiológicas e comportamentais já citadas anteriormente, tanto nos bebês quanto nos funcionários (AURÉLIO; TOCHETTO, 2010b; ZAMBERLAN et al., 2008).

Em estudo realizado na cidade de Fortaleza - CE, os valores do jato d'agua da torneira para lavagem das mãos foram semelhantes ao do presente estudo, variando de 72,4 a 80,4 dB (CARDOSO; CHAVES; BEZERRA, 2010).

Para tentar minimizar os ruídos intensos podese encapar as rodas da mesa de inox com silicone, diminuir a intensidade do jato d'água na torneira, regular os alarmes dos aparelhos e atendê-los prontamente, colocar silicone ou borracha na tampa do (cesto de) lixo para diminuir o impacto em seu fechamento, abrir e fechar as portinholas da incubadora com cuidado, sempre segurandoas ao abrir, e empurrando-as ao fechar, e retirar o papel dos dispensadores com maior cuidado e delicadeza (ZAMBERLAN, 2006).

A percepção da maioria das profissionais entrevistadas é de que a UN é um local ruidoso, inclusive alguns referiram que estes ruídos chegam a causar prejuízos na sua rotina de 
trabalho e também interferem na sua vida fora deste ambiente.

Estudo realizado em um Hospital Universitário na cidade de Santa Maria - RS objetivou conhecer a percepção de profissionais que atuavam na UTIN e também de pais sobre os ruídos presentes nesta unidade, além de mensurar esses níveis para uma posterior comparação das duas vertentes da pesquisa. O referido estudo apontou que 97,7\% dos profissionais entrevistados afirmaram que os ruídos podem provocar alterações nos indivíduos, e apontaram a irritabilidade e o estresse como os maiores efeitos prejudiciais para os profissionais, confirmando que eles têm consciência sobre os prejuízos causados para os mesmos no ambiente de trabalho e também fora dele (AURÉLIO; TOCHETTO, 2010b).

Em um estudo desenvolvido na cidade de Ribeirão Preto - SP (SILVA et al., 2012), que teve por objetivo conhecer a percepção da equipe que atuava na UCIN sobre o ruído ambiental, apontou que as principais fontes citadas pelos profissionais foram as mesmas encontradas no presente estudo, confirmando que não somente na unidade estudada, mas profissionais de outros serviços também reconheceram as mesmas fontes como (as) mais ruidosas.

As respostas dos profissionais em relação aos efeitos do ruído nos RNs estão em conformidade com a literatura. O excesso desse pode causar no RN: alteração auditiva, alteração do ciclo sono/ vigília, alterações comportamentais, estresse e irritabilidade, taquicardia, queda de saturação e desorganização (AURÉLIO; TOCHETTO, 2010b; SILVA et al., 2012).

O ambiente extrauterino é prejudicial para o desenvolvimento neuropsicomotor dos RNs, e um ambiente ruidoso, com luminosidade e manipulação em excesso, é ainda pior. Ruído em demasia provoca interrupções constantes no sono do bebê, o qual é fundamental para a maturação cerebral, interfere no ganho (de) peso, presença de irritabilidade e choro. Quanto mais próximo do ambiente fetal for a unidade, maior a qualidade de desenvolvimento oferecida aos bebês (ZAMBERLAN, 2006; ZAMBERLAN et al., 2008).

Níveis de Leq mais altos foram encontrados em estudo realizado em São Paulo, onde durante quatro dias de coleta obteve Leq de $64 \mathrm{~dB}$ (KAKEHASHI et al., 2007). Também foram mais altos em estudo desenvolvido na cidade de Ribeirão Preto, onde encontrou Leq de 60,8 dB (ZAMBERLAN et al., 2008). Estudo realizado no Rio Grande do Sul, mensurou-se os ruídos produzidos nas 24 horas, durante nove dias, em quatro locais diferentes (uma sala de cuidados intensivos, uma sala de cuidados intermediários, um isolamento e corredor da unidade), também encontrou um Leq maior, variando de 58,5 dB a 65,8 dB (AURÉLIO; TOCHETTO, 2010a).

A unidade pesquisada alcançou um nível médio de ruído dentro do recomendado pela $A B N T$, sendo $45 \mathrm{~dB}$ o valor aceitável. Porém, foram obtidos picos muito altos de Lpeak e Lmáx, níveis acima do limiar de dor, que se encontra em torno de 130 dB (CARVALHO; PEDREIRA; AGUIAR, 2005). O maior Lpeak, 144,8 dB, foi verificado no período vespertino, no sexto dia de coleta, porém, não houve nenhum registro no diário de campo neste dia para identificação específica da fonte do ruído.

Durante a primeira semana de coleta, no período matutino, uma das pesquisadoras fez um diário de campo, através de observação das fontes e situações de ruídos mais intensos. A seguir, serão apresentadas algumas destas situações observadas.

Por exemplo, durante o disparo do alarme de um oxímetro de pulso, observou-se uma variação entre $89,7 \mathrm{~dB}-91,1 \mathrm{~dB}$, e neste mesmo dia, o pico foi de $121,2 \mathrm{~dB}$, no período matutino, porém, não se sabe o que ocasionou nível tão elevado. Estudo realizado em duas salas de UTIN de um Hospital Universitário de São Paulo constatou que uma das 
principais fontes de ruído foi o disparo de alarmes de equipamentos (PEIXOTO et al., 2011). Apesar de serem itens necessários para monitorização dos bebês em tratamento, existem alternativas para minimizar essa situação, como o ajuste de volume máximo aceitável e alarmes luminosos para diminuir o ruído.

A pesagem dos bebês era realizada três vezes por semana, e a balança transportada de um lado para o outro da unidade por meio de uma mesa móvel. Os níveis variaram de 88,6 dB - $105 \mathrm{~dB}$ durante tal evento. No quarto dia aconteceu a lavagem de uma incubadora no interior da unidade, que não dispunha de um local adequado (para) sua realização. Durante este procedimento, os níveis de pressão sonora variaram de 89,5 dB a 102,1 dB.

As alternativas sugeridas pelos próprios profissionais para o controle de ruídos foram muito pertinentes e demonstraram o reconhecimento da necessidade de instituir medidas para o aprimoramento do nível de ruídos na unidade, através da manutenção periódica dos aparelhos (incubadoras, oxímetros), conscientização da equipe, diminuição do tom de voz, mudanças nas lixeiras, diminuição do volume dos alarmes, restrição do acesso à unidade, local próprio para lavagem de incubadora, manutenção das rodas dos objetos emborrachados, ajuste do jato de água da torneira, manter desligada a umidade quando o reservatório estiver sem água.

Uma nova UN foi construída na instituição do presente estudo, e alguns problemas atualmente encontrados, como a falta de local adequado para lavagem de incubadoras, em um curto período de tempo, estarão resolvidos. Enquanto isso é necessária certa atenção e cuidado por parte da equipe para diminuir os picos intensos e elevados de ruídos, uma vez que as respostas obtidas pelos funcionários no questionário evidenciaram ciência sobre os motivos e prejuízos do ruído. Apesar de o nível médio encontrado estar dentro do recomendado, espera-se o aprimoramento dos ruídos que interferem principalmente no sono do bebê, podendo causar assim um atraso no seu desenvolvimento.

Alguns benefícios da reduçãodoruídona UN para o bebê/família incluem: aumento na estabilidade fisiológica, melhora na taxa de crescimento, maturação neurossensorial mais consistente e apropriada para a idade e menos problemas, a longo prazo, nas áreas de processamento auditivo, fala e linguagem (BRASIL, 2011).

Estudos descritivos que permitem a revelação diagnóstica de um contexto local podem sugerir problemática semelhante em outras UNs, considerando quemuitos dos problemas encontrados neste estudo são semelhantes em outros locais. Esse estudo trouxe vários apontamentos observados pelos profissionais que podem contribuir para a elaboração de um protocolo de controle de ruídos, em uma tentativa de minimizar valores elevados dos mesmos. A partir da elaboração de protocolos, sugere-se estudos de intervenção para verificação de sua eficácia e modificação da prática.

\section{Conclusão}

Ao final do estudo pode-se concluir que os trabalhadores da UN consideraram a unidade ruidosa e demonstraram ciência de que esses ruídos podem interferir tanto no desenvolvimento do bebê internado, como também na sua rotina de trabalho e vida pessoal.

As fontes de ruído encontradas foram: alarmes de equipamentos, conversas paralelas, telefone, lixeiras, objetos arrastados, intercorrências, fluxo intenso de pessoas, lavagem da incubadora, portinholas da incubadora, válvula redutora de pressão, bomba de infusão, gaveta do carrinho de emergência.

Constatou-se também que o valor médio dos ruídos encontrados, em decibéis, está dentro do preconizado pela ABNT, porém foram encontrados níveis superiores aos recomendados de Lpeak e Lmax. 


\section{Referências}

ALS, H. Toward a synactive theory of development: promise for the assessment and support of infant individuality. Infant Mental Health Journal, New York, v. 3, n. 4, p. 229-243, 1982.

ASSOCIAÇÃO BRASILEIRA DE NORMAS TÉCNICAS - ABNT. NBR 10151: acústica avaliação do ruído em áreas habitadas, visando o conforto da comunidade - procedimento. Rio de Janeiro: ABNT, 2000.

ASSOCIAÇÃO BRASILEIRA DE NORMAS TÉCNICAS - ABNT. NBR 10152: níveis de ruídos para conforto acústico. Rio de Janeiro: ABNT, 1987.

AURÉLIO, F. S.; TOCHETTO, T. M. Mensuração do ruído em uma unidade de terapia intensiva neonatal. Acta Pediátrica Portuguesa, Lisboa, v. 41, n. 2, p. 64-68, 2010a.

AURÉLIO, F. S.; TOCHETTO, T. M. Ruído em uma unidade de terapia intensiva neonatal: mensuração e percepção de profissionais e pais. Revista Paulista de Pediatria, São Paulo, v. 28, n. 2, p. 162-160, 2010 b.

BRASIL. Ministério da Saúde. Atenção humanizada ao recém-nascido de baixo peso: método canguru. Brasília, 2011.

BYERS, J. F. Components of developmental care and the evidence for their use in the NICU. MCN The American Journal of Maternal/Child Nursing, New York, v. 28, n. 3, p. 174-180, 2003.

CARDOSO, M. V. L. M. L.; CHAVES, E. M. C.; BEZERRA, M. G. A. Ruídos e barulhos na unidade neonatal. Revista Brasileira de Enfermagem, Brasília, v. 63 , n. 4 , p. 561-566, 2010.

CARVALHO, W. B.; PEDREIRA, M. L. G.; AGUIAR, M. A. L. Nível de ruídos em uma unidade de cuidados intensivos pediátricos. Jornal de Pediatria, Porto Alegre, v. 81, n. 6, p. 495-498, 2005.

GAÍVA, M. A. M.; MARQUESI, M. C.; ROSA, M. K. O. O sono do recém-nascido internado em unidade de terapia intensiva: cuidados de enfermagem. Ciência, Cuidado e Saúde, Maringá, v. 9, n. 3, p. 602609, 2010.
KAKEHASHI, T. Y.; PINHEIRO, E. M.; PIZZARRO, G.; GUILHERME, A. Noise level in neonatal intensive care unit. Acta Paulista de Enfermagem, São Paulo, v. 20, n. 4, p. 404-409, 2007.

PEIXOTO, P. V.; ARAÚJO, M. A. N.; KAKEHASHI, T. Y.; PINHEIRO, E. M. Nível de pressão sonora em unidade de terapia intensiva neonatal. Revista da Escola de Enfermagem da USP, São Paulo, v. 45, n. 6, p. 1309-1314, 2011.

REICHERT, A. P. S.; LINS, R. N. P.; COLLET, N. Humanização do cuidado da UTI neonatal. Revista Eletrônica de Enfermagem, Goiânia, v. 9, n. 1, p. 200-213, 2007. Disponível em: <http://www.fen. ufg.br/revista/v9/n1/v9n1a16.htm>. Acesso em: 31 jun. 2014.

RODARTE, M. D. O.; SCOCHI, C. G. S.; LEITE, A. M.; FUJINAGA, C. I.; ZAMBERLAN, N. E.; CASTRAL, T. C. O ruído gerado durante a manipulação das incubadoras: implicações para o cuidado de enfermagem. Revista LatinoAmericana de Enfermagem, Ribeirão Preto, v. 13, n. 1, p. 79-85, 2005.

SANTOS, L. M.; RIBEIRO, I. S.; SANTANA, R. C. B. Identificação e tratamento da dor no recém-nascido prematuro na Unidade de Terapia Intensiva. Revista Brasileira de Enfermagem, Brasília, v. 65, n. 2, p. 269-275, 2012.

SILVA, A. C. A.; ZAMBERLAN-AMORIM, N. E.; FONSECA, L. M. M.; FUJINAGA, C. I.; BENUTTI, D. P.; SCOCHI, C. G. S. Percepção da equipe multiprofissional sobre o ruído em unidade de cuidado intermediário neonatal. Acta Paulista de Enfermagem, São Paulo, v. 25, n. 1, p. 74-79, 2012.

TAMEZ, R. N.; SILVA, M. P. J. Enfermagem na UTI neonatal: assistência ao recém-nascido de alto risco. 5. ed. Rio de Janeiro: Guanabara Koogan, 2013.

WORLD HEALTH ORGANIZATION - WHO. Guidelines for community noise. 1999. Disponível em: <http://www.who.int/docstore/peh/noise/ Commnoise4.html>. Acesso em: 31 jun. 2012. 
ZAMBERLAN, N. E. Ruido na unidade de cuidado intermediário neonatal de um hospital universitário de Ribeirão Preto - SP. 2006. $102 \mathrm{f}$. Dissertação (Mestrado em Enfermagem em Saúde Pública) - Escola de Enfermagem de Ribeirão Preto, Universidade de São Paulo, Ribeirão Preto, 2006.

ZAMBERLAN, N. E.; ICHISATO, S. M. T.; RODARTE, M. D. O.; FUJINAGA, C. I.; HASS, V. J.; SCOCHI, C. G. S. Ruído na unidade de cuidado intermediário neonatal de um hospital universitário. Ciência, Cuidado e Saúde, Maringá, v. 7, n. 4, p. 431-438, 2008. 